\title{
Sergey I. Repin, Stefan A. Sauter: "Accuracy of Mathematical Models"
}

\author{
EMS, 2020, 317 pp.
}

\section{Thomas Wick ${ }^{1}$}

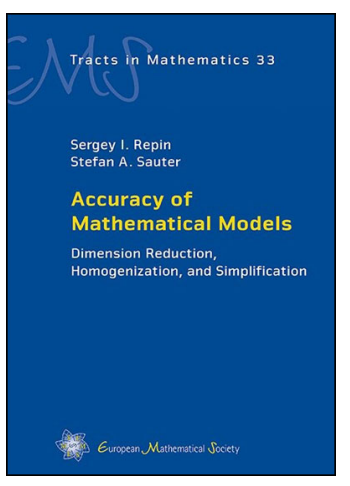

The book by Repin and Sauter is an inspiring piece of work. The key question is centered around 'How accurate is a mathematical model?' by deriving model error estimates arising in different approaches for treating partial differential equations and variational problems of elliptic type.

Accuracy is nothing else than measuring distances, in metric spaces or normed vector spaces, between (unknown) exact solutions and their (numerical) approximations in terms of error estimates. There is a rich theory on complete metric spaces and Banach spaces which forms the basis for some well-posedness results and numerical analysis of partial differential equations and variational problems. While the theory for linear (elliptic) problems is fairly complete, nonlinear (time-dependent) differential equations and variational inequality systems are subject of ongoing research.

Error estimates often include several sources such as model-, regularization-, homogenization-, discretization-, interpolation-, linear solver-, nonlinear solver-, quadrature-, and implementation (bugs) errors. But also the exact solution to compare with, might be error-prone from inaccurate experimental setups, uncertain data, and measurement errors.

The important confession one has to make is the fact that such errors never can be avoided. Rather, the aim is to design error estimates that allow us to quantify their influence and to adjust those terms with the largest contributions.

T. Wick

thomas.wick@ifam.uni-hannover.de

1 Hannover, Germany 
As an illustration let us consider the spaces $(X,\|\cdot\|)$ and $(\tilde{X},\|\cdot\|)$. Therein, $u \in X$ and $\tilde{u} \in \tilde{X}$ are considered as points describing the exact solution on the continuous level and its approximation, respectively. An error estimator is usually denoted by a positive $\eta$. To estimate the distance (i.e., the accuracy) between $u$ and $\tilde{u}$, the objective is to establish two-sided estimates of the form

$$
c_{1} \eta \leq\|u-\tilde{u}\| \leq c_{2} \eta
$$

with some positive constants $c_{1}, c_{2}$. The upper and lower bounds are known as reliability and efficiency of the error estimator, respectively. Asymptotically, we aim for

$$
\|u-\tilde{u}\| \rightarrow 0 .
$$

From a practical viewpoint in computations, we are satisfied when

$$
\|u-\tilde{u}\| \leq c_{2} \eta \leq T O L,
$$

where TOL is some given positive small tolerance, but larger than the machine precision of the computer. When various error sources are present, the triangle inequality is used to split them into different parts. An example in the book at hand is provided in the chapter on homogenization. Let $\Omega$ be a bounded Lipschitz domain in $\mathbb{R}^{n}$, where $n$ is the dimension. Moreover, let $H_{0}^{1}(\Omega)$ be the usual Sobolev space of square-integrable functions with one weak square-integrable derivative and vanishing on the boundary. For periodic heterogeneities of the (small) lengthscale $\varepsilon \in(0,1]$, the weak form of the homogenization problem reads: Find $u_{\varepsilon} \in H_{0}^{1}(\Omega)$ such that

$$
a_{\varepsilon}\left(u_{\varepsilon}, \varphi\right)=l(\varphi) \quad \text { for all } \varphi \in H_{0}^{1}(\Omega),
$$

where

$$
\begin{aligned}
a_{\varepsilon}\left(u_{\varepsilon}, \varphi\right) & =\int_{\Omega} A_{\varepsilon} \nabla u \cdot \nabla \varphi d x+\int_{\Omega}\left(b_{\varepsilon} \cdot \nabla u+c_{\varepsilon} u\right) \varphi d x, \\
l(\varphi) & =\int_{\Omega} f \varphi d x .
\end{aligned}
$$

Here, $A_{\varepsilon}$ is a symmetric diffusion matrix with coefficients in $L^{\infty}$, the vector $b_{\varepsilon} \in$ $W^{1, \infty}\left(\Omega, \mathbb{R}^{n}\right)$ is a convection term and $c_{\epsilon} \in L^{\infty}\left(\Omega, \mathbb{R}^{n}\right)$ is a reaction term. In many applications (see e.g., [2] for a concise overview and literature references) periodic structures play an important role. Here, the effective material properties $A_{\varepsilon}, b_{\varepsilon}$ and $c_{\epsilon}$ are derived on a reference cell.

From a numerical viewpoint, solving the previous problem with any method requires too much effort when $\varepsilon$ is very small. The reason is that $\varepsilon$ must be resolved by the discretization resulting into large systems of linear equations. Using homogenization the solution is averaged, which is based on asymptotic expansions of the form

$$
u_{\varepsilon}=\sum_{i=0}^{\infty} \varepsilon^{i} u_{i}
$$


Therein, $u_{0}$ is the zero-order approximation, $u_{1}$ the first-order approximation, and so forth. The infinite-dimensional solutions can be approximated (i.e., discretized) with a Galerkin method (for instance Galerkin finite elements) denoted by $\tilde{u}_{\varepsilon}$. In the following, let us consider in more detail the first-order approximation $u_{1, \varepsilon}:=$ $u_{0}+\varepsilon u_{1}$ and its discrete representation $\tilde{u}_{1, \varepsilon}$. The total error of interest is

$$
E_{t o t}:=\left\|\nabla\left(u_{\varepsilon}-\tilde{u}_{1, \varepsilon}\right)\right\| .
$$

In order to identify the dominant error source, the continuous-level first-order approximation $u_{1, \varepsilon}$ is inserted and it holds with the triangle inequality:

$$
\begin{aligned}
E_{\text {tot }} & :=\left\|\nabla\left(u_{\varepsilon}-u_{1, \varepsilon}+u_{1, \varepsilon}-\tilde{u}_{1, \varepsilon}\right)\right\| \leq\left\|\nabla\left(u_{\varepsilon}-u_{1, \varepsilon}\right)\right\|+\left\|\nabla\left(u_{1, \varepsilon}-\tilde{u}_{1, \varepsilon}\right)\right\| \\
& =: E_{\text {hom }}+E_{\text {approx }} .
\end{aligned}
$$

The first part $E_{\text {hom }}$ is the error induced by the homogenization, while the second part $E_{\text {approx }}$ is the approximation error due to the discretization. At this point, the goals are two-fold. The first objective is to clarify whether the error parts are computable. The second aim is to identify the dominating error contribution. Concerning the first question, the homogenization error $E_{\text {hom }}$ is not computable because the approximation $u_{1, \varepsilon}$ is not known, while $E_{\text {approx }}$ can be bounded with available information obtained within the discretization. To this end, Repin and Sauter further find that the approximation error can be bounded as follows:

$$
E_{\text {approx }} \leq \eta:=\rho_{1} \epsilon+\rho_{2} \delta+\rho_{3} \delta_{\text {int }}+\rho_{4} \hat{\delta}+\tau_{1}+\tau_{2}+\tau_{3} .
$$

These seven error parts are further discussed in the book. Ideally such estimates are confirmed by prototype numerical simulations.

Typical further questions are the choice of the norm $\|\cdot\|$ and the resulting convergence order in terms of adjustable parameters for approximating $u$ with a sequence $\left\{\tilde{u}_{l}\right\}_{l \in \mathbb{N}}$. Depending on regularity properties, the geometry, smoothness of functions and coefficients, appropriate Sobolev spaces can be chosen. Hence various norms may be available yielding different convergence orders.

Error estimates can be classified into two categories: a priori and a posteriori error estimates. The former incorporate the (unknown) exact solution $u$, such that $\eta:=\eta(u)$, and yield qualitative convergence rates for asymptotic limits. They can be derived before (thus a priori) the approximation $\tilde{u}$ is known. On the other hand, a posteriori error estimates of the form $\eta:=\eta(\tilde{u})$ explicitly employ the approximation $\tilde{u}$ and therefore yield quantitative information with computable majorants (i.e., bounds) and can be further utilized to design adaptive schemes. An example (among others) is provided for modeling-discretization strategies in Sect. 5.5.3 (pages 230ff) in the book at hand.

For computable (a posteriori) error estimates $\eta:=\eta(\tilde{u})$, it is furthermore interesting to evaluate the (reciprocal) effectivity index

$$
I_{e f f}=\frac{\eta}{\|u-\tilde{u}\|}
$$

in order to judge the approximation quality. This can be done for known exact solutions $u$, derived for prototype problems. Alternatively, reference solutions $u$ might 
be obtained purely based on numerical (brute-force) simulations in which the discretization parameters are usually chosen sufficiently small. Asymptotically, in either attempt, the aim is to achieve $I_{\text {eff }} \rightarrow 1$.

Repin and Sauter summarize error estimates for several state-of-the-art developments for dimension reduction models (Chap. 3), model simplifications (Chap. 4), elliptic homogenization (Chap. 5) and model conversions (Chap. 6). In each chapter, comments on modeling-discretization strategies are made, which are useful for computer implementations and numerical simulations. In the preface of the book the main motivations and goals are clearly described. Therein also the need to restrict to certain concepts and errors, mainly model errors, are justified. Discretization errors are considered as a special class of modeling errors.

Chapter 1 summarizes classical inequalities of Friedrichs, Poincaré, and Korn in order to provide a self-contained work. Chapter 2 provides estimates to measure the distance to exact solutions. In Theorem 2.2.1 the main error identity is established. Afterwards, several examples of linear problems and further applications are discussed in great detail. In Sect. 2.6, errors due to numerical approximations such as discretization errors based on a posteriori estimates of functional type are discussed.

The key results are contained in Chaps. 3-6. First, Repin and Sauter concentrate on dimension reduction models. These are well-known in continuum mechanics for solids and fluids. For the former, elastic thin-walled structures can be derived. For fluids, a well-known example is Reynolds lubrication equation, which can be obtained by upscaling the incompressible Navier-Stokes equations.

In Chap. 4, model simplifications are addressed. Here, the emphasis is on simplifications of coefficients such as material parameters. In practice these may be tensorvalued, heterogeneous, non-isotropic, and possibly non-smooth. Often in mathematical modeling they are assumed to be single-valued, homogeneous, isotropic and smooth. An example is the Saint-Venant Kirchhoff material in solid mechanics in which we only deal with two independent parameters: the so-called Lamé coefficients. These can be linked to Poisson's ratio (behavior of the material under compression) and Young's modulus measuring the stiffness of a solid material. The book at hand provides estimates to measure the deviation between complicated coefficients and their simpler counterparts. Next, simplifications of boundary conditions are discussed. An exciting part is then Sect. 4.4 (Comments), where rough coefficients employing higher-order estimates in Sobolev spaces, modeling-discretization adaptivity, and uncertain data are briefly referenced by pointing to numerous references in the literature. In Chap. 5, elliptic homogenization is addressed, which is a prominent topic and was briefly introduced before. Again, error estimates yielding computable majorants are provided. Similar to the previous chapter, the 'Comments' part (Sect. 5.6) is recommended. First, specific regularity constants are provided. Second, modelingdiscretization techniques for adaptive strategies are derived. Finally, references to multiscale problems are provided.

While the book by Repin and Sauter uses functional-type estimates [9], concurrent developments by others in a posteriori error estimation and adaptivity are residualbased techniques [11], error estimates for contact problems [13], goal functionals (i.e., technical quantities of interest) [1, 3, 4], flux reconstructions [7], and adaptive multiscale predictive modeling [8]. Moreover, axioms of adaptivity were proposed in 
[5], and they help to establish proofs of optimal convergence rates for certain adaptive algorithms.

For general goal functionals $[1,3,4,8]$ of the form $J: X \rightarrow \mathbb{R}$, it should be noticed that these are not necessarily norms anymore. In fact, $J(u) \in \mathbb{R}$ and $J(\tilde{u}) \in \mathbb{R}$ may have negative values, and $J(u)=J(\tilde{u})$ may happen for $u \neq \tilde{u}$. This is a drawback jeopardizing rigorous error estimates. On the other hand, such goal functionals allow for problem-specific error quantifications in which not the entire solution is measured in a global norm, but only parts of the solution or derivative information evaluated in subdomains, boundaries, interfaces or single points. Typical examples are mean value evaluations in the domain $\Omega$, derivative information over interfaces $\Gamma$, or point value evaluations, respectively:

$$
J(u)=\int_{\Omega} u d x, \quad J(u)=\int_{\Gamma} \partial_{n} u d s, \quad J(u)=u(x), x \in \Omega .
$$

In fluid and solid mechanics, stress evaluations are often of interest:

$$
J(u)=\int_{\Gamma} \sigma_{s}(u) n e d s, \quad J(v, p)=\int_{\Gamma} \sigma_{f}(v, p) n e d s,
$$

where $\sigma_{s}(u)$ is the solid Cauchy stress tensor with vector-valued displacements $u: \Omega \rightarrow \mathbb{R}^{n}$, and $\sigma_{f}(v, p)$ is the fluid Cauchy stress tensor with the vector-valued velocity $v: \Omega \rightarrow \mathbb{R}^{n}$ and scalar-valued pressure $p: \Omega \rightarrow \mathbb{R}$. Furthermore, $e$ is a unit vector in $\mathbb{R}^{n}$. The last goal functional is important in aerodynamics for computing drag (with $e=(1,0,0)^{T}$ as the main flow direction) and lift values (with $e=(0,1,0)^{T}$ orthogonal to the main flow direction), e.g. for airplanes. As for norms, the objective is to derive two-sided estimates

$$
c_{1} \eta \leq|J(u)-J(\tilde{u})| \leq c_{2} \eta
$$

Since $|J(u)-J(\tilde{u})|=0$ may happen, the lower bound estimate remains a questionable task and was only recently achieved in [6] using a so-called saturation assumption.

Findings as discussed in this book are useful for two research directions of equal importance. First, an increasing number of mathematical methods and numerical techniques require a careful rigorous numerical analysis for which suggestions can be obtained from this book. Second, there is on the other hand, an increasing number of models and numerical techniques in scientific computing and computational engineering addressing challenging variational multiphysics problems. These are characterized as nonstationary, nonlinear, coupled systems of partial differential equations. Examples are for instance fluid-structure interaction [10] and coupled variational inequality systems [12]. Currently, complete analyses for such problems are out of scope. Partial results include goal-oriented error estimation for fluid-structure interaction and multigoal-oriented error estimates (i.e., controlling simultaneously different goal functionals) with balancing of discretization and nonlinear iteration errors, respectively. Despite these developments, there is a current need to also employ (or couple with) techniques as described by Repin and Sauter, that may help to design 
homogenized approaches, model simplifications, adaptive schemes, and model conversions that are cheaper in their numerical solution without deteriorating the mathematical accuracy.

Summarizing, the book at hand is a valuable reference for state-of-the-art results on model error estimates for partial differential equations and variational problems of elliptic type by covering various approaches that can usually not be found in one single work. It should be appealing to graduate students with prerequisites in mathematical modeling, differential equations, continuum mechanics, and homogenization as well as advanced researchers who are interested in employing accuracy concepts in function spaces. Therefore, this book is an inspiring source for both advanced teaching and conducting research in the fascinating field of numerical error analysis.

Funding Note Open Access funding enabled and organized by Projekt DEAL.

Publisher's Note Springer Nature remains neutral with regard to jurisdictional claims in published maps and institutional affiliations.

Open Access This article is licensed under a Creative Commons Attribution 4.0 International License, which permits use, sharing, adaptation, distribution and reproduction in any medium or format, as long as you give appropriate credit to the original author(s) and the source, provide a link to the Creative Commons licence, and indicate if changes were made. The images or other third party material in this article are included in the article's Creative Commons licence, unless indicated otherwise in a credit line to the material. If material is not included in the article's Creative Commons licence and your intended use is not permitted by statutory regulation or exceeds the permitted use, you will need to obtain permission directly from the copyright holder. To view a copy of this licence, visit http://creativecommons.org/licenses/by/4.0/.

\section{References}

1. Ainsworth, M., Oden, J.T.: A Posteriori Error Estimation in Finite Element Analysis. Pure and Applied Mathematics (New York). Wiley-Interscience, New York (2000)

2. Allaire, G.: Shape Optimization by the Homogenization Method. Springer, Berlin (2002)

3. Bangerth, W., Rannacher, R.: Adaptive Finite Element Methods for Differential Equations. Lectures in Mathematics. Birkhäuser, ETH Zürich (2003)

4. Becker, R., Rannacher, R.: An optimal control approach to a posteriori error estimation in finite element methods. In: Acta Numerica, pp. 1-102. Cambridge University Press, Cambridge (2001)

5. Carstensen, C., Feischl, M., Page, M., Praetorius, D.: Axioms of adaptivity. Comput. Math. Appl. 67(6), 1195-1253 (2014)

6. Endtmayer, B., Langer, U., Wick, T.: Two-side a posteriori error estimates for the dual-weighted residual method. SIAM J. Sci. Comput. 42(1), A371-A394 (2020)

7. Ern, A., Vohralík, M.: Adaptive inexact Newton methods with a posteriori stopping criteria for nonlinear diffusion PDEs. SIAM J. Sci. Comput. 35(4), A1761-A1791 (2013)

8. Oden, J.T.: Adaptive multiscale predictive modelling. Acta Numer. 27, 353-450 (2018)

9. Repin, S.: A Posteriori Estimates for Partial Differential Equations. Radon Series on Computational and Applied Mathematics, vol. 4. de Gruyter, Berlin (2008)

10. Richter, T.: Fluid-Structure Interactions: Models, Analysis, and Finite Elements. Springer, Berlin (2017)

11. Verfürth, R.: A Posteriori Error Estimation Techniques for Finite Element Methods. Oxford University Press, London (2013)

12. Wick, T.: Multiphysics Phase-Field Fracture: Modeling, Adaptive Discretizations, and Solvers. Radon Series on Computational and Applied Mathematics, vol. 28. de Gruyter, Berlin (2020)

13. Wohlmuth, B.: Variationally consistent discretization schemes and numerical algorithms for contact problems. Acta Numer. 20, 569-734 (2011) 